\title{
Helios G4: Pushing the Limits of TEM Sample Preparation and STEM Resolution
}

\author{
Tomáš Vystavěl ${ }^{1}$, Lubomír Tůma ${ }^{1}$, Jan Skalický1 ${ }^{1}$, Richard Young ${ }^{2}$ \\ ${ }^{1}$ FEI Company, Vlastimila Pecha 12, Brno, Czech Republic \\ ${ }^{2}$ FEI Company, 5350 NE Dawson Creek Drive, Hillsboro, OR, USA
}

Shrinking sizes of observed features and advancements in (S)TEM techniques requires higher quality TEM sample preparation as well as extended low-kV STEM-in-SEM analysis. In this contribution we present a new generation of the FEI Helios family, configured for ultimate sample preparation and improved STEM-in-SEM imaging. The main improvements to the system can be summarized in sample handling, improved electron and ion optics, and in the introduction of a brand new workflow combining advanced TEM sample preparation and high-resolution STEM imaging in a single instrument.

Common workflows include top-down, plan view, and inverted lamellas as well as tomography sample preparation. Sample handling is achieved by a combination of the well-established piezo stage to manipulate and observe bulk samples in combination with newly developed TEM-like rod for sample thinning and STEM imaging. The dual-tilt rod has a long tilt axis perpendicular to the electron and ion columns and second axis allowing sample flip. Opening of the rod from one side facilitates sample milling and cleaning using FIB, and enables optimized signal collection by EDS. The sample rod can be automatically inserting and retracting into the specimen chamber, permitting automated switching from bulk stage to STEM operation. Sample lift-out is achieved by the EasyLift ${ }^{\mathrm{TM}}$ manipulator.

The focused ion beam (FIB) is an established and powerful technology for site-specific sample preparation. The newly developed Phoenix FIB column brings significant improvement over all existing columns by achieving the possibility to precisely position the low energy ion beam during final lamella thinning and cleaning at 500eV (Figure 1c), while maintaining high milling rates at high energies. The column allows operators to create site specific ultra-thin lamellas with damage below $1 \mathrm{~nm}$ and maximum cut fidelity.

The improved SEM/STEM is based on the FEI Elstar column; including a Schottky field emission electron gun with improved monochromator performance allows usage the monochromated beam at higher beam currents for improved low-energy imaging. In order to improve the STEM performance the system is equipped with a retractable second (lower) pole piece in such a way that the system can be operated both as a traditional SEM in a FIB/SEM configurations as well in a high-resolution in-lens SEM/STEM mode (Figure 1a,b). The lower part of the STEM objective is equipped with an integrated solid state segmented STEM. The presented developments enable a significant improvement in the STEM resolution of the original system by a factor of two, with the possibility to simultaneously acquire various SEM and STEM signals to provide surface information as well as structural and compositional information as demonstrated on the Figure 2.

We are able to demonstrate resolution of lattice planes on several materials such as carbon nanotubes $(0.34 \mathrm{~nm}$ at $20-30 \mathrm{kV})$ or Tungsten Disulfide $(0.27 \mathrm{~nm}$ at $30 \mathrm{kV})$. Configurability of the system opens the space for in-situ experiments, improving existing workflows and expanding possibilities to bring new approaches in materials characterization. 


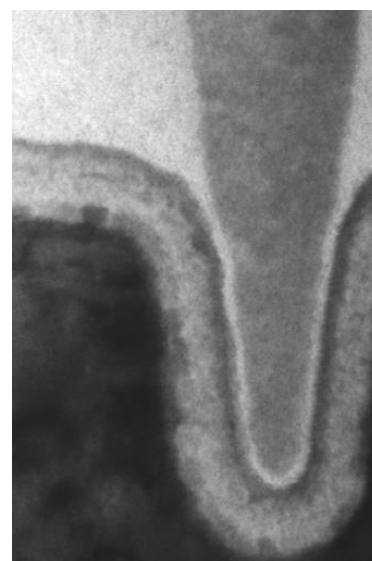

a

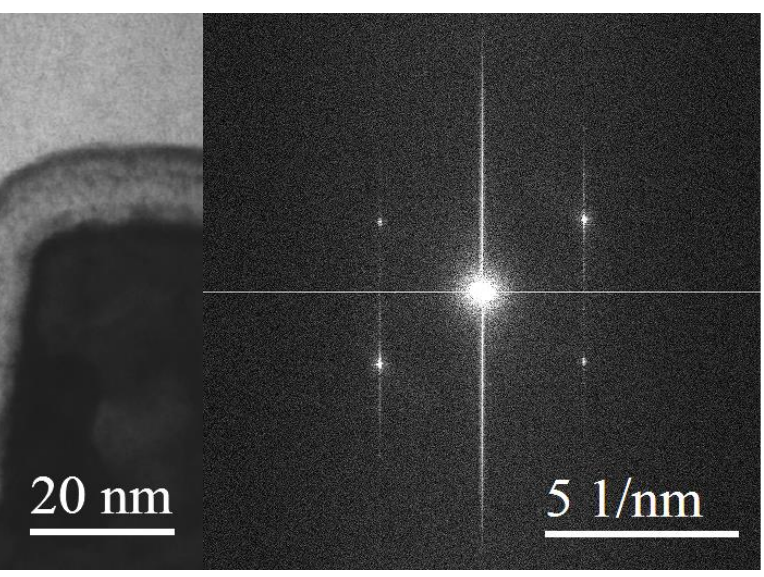

b

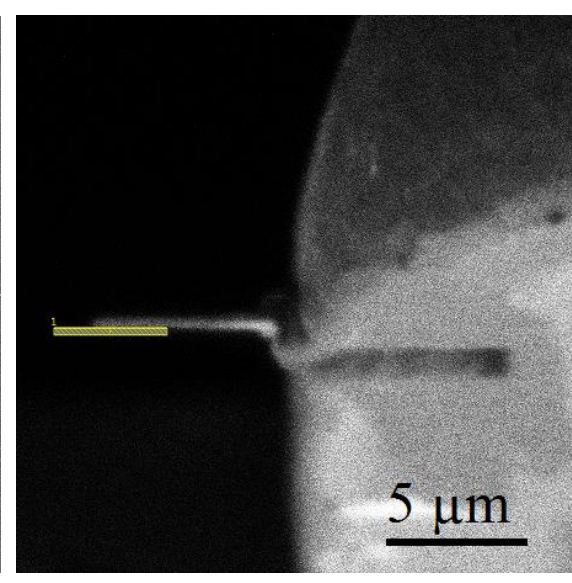

C

Figure 1 a) typical BF STEM image of a semiconductor fin finished with low kV cleaning, (b) FFT from silicon region taken from higher magnification picture, $\{111\}$ planes are clearly resolved, (c) ioninduced secondary electron image of a lamella on the grid taken at $500 \mathrm{~V}$ during its final cleaning demonstrating easiness of the pattern placement during final FIB cleaning.
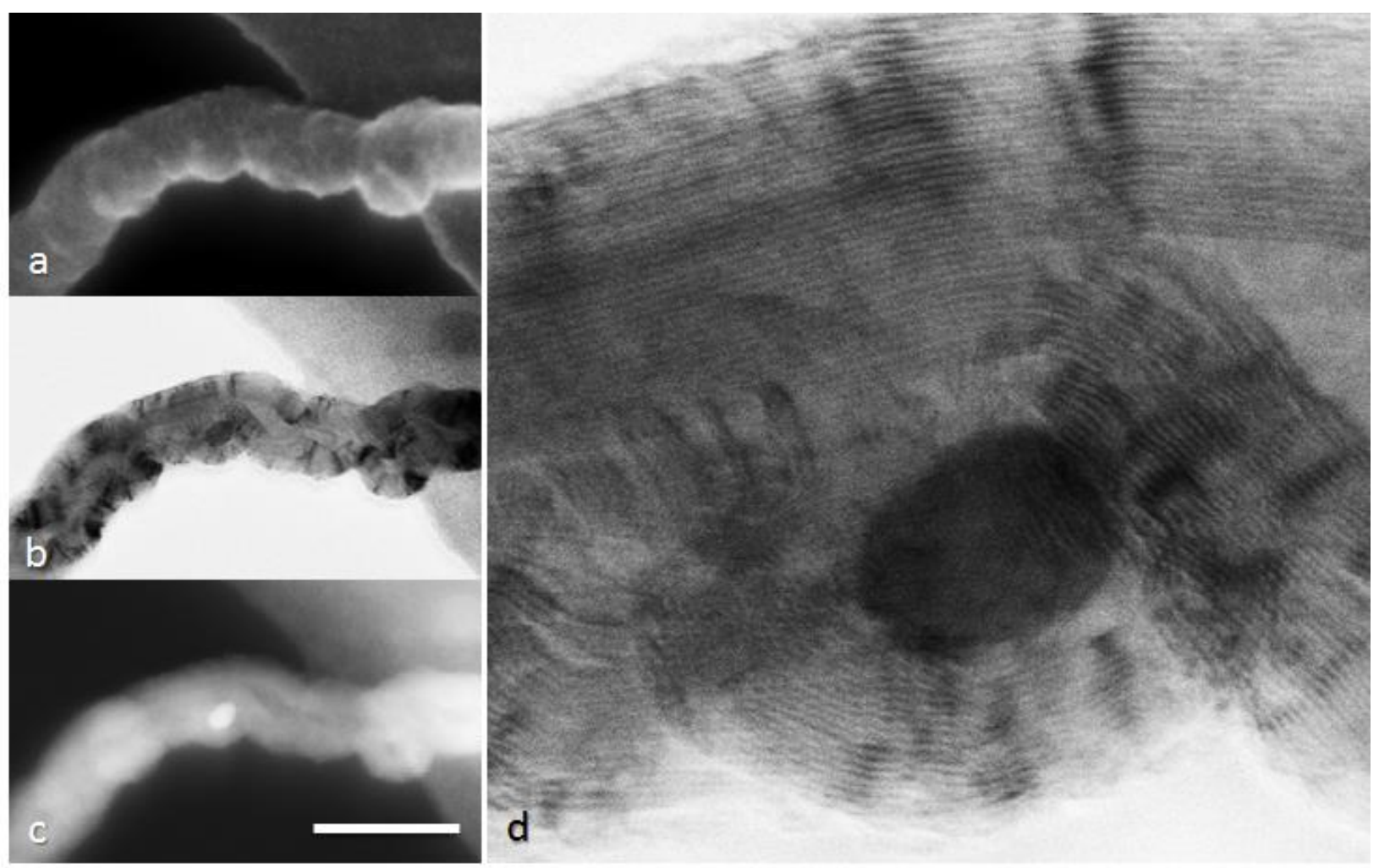

Figure 2 G4 imaging of a multiwall CNT at 30kV showing (a) secondary electron image, (b, d) bright field STEM and (c) HAADF image. Simultaneous imaging shows surface details on secondary electron image (a), structural information on STEM BF (b) and compositional information on STEM HAADF note catalytic particle is easily resolved on HAADF, the scale bar represents $50 \mathrm{~nm}$. 